\title{
THE MARCH MEETING OF THE CHICAGO SECTION.
}

THE twenty-first regular meeting of the Chicago Section of the American Mathematical Society was held at the University of Chicago, on Saturday, March 30, 1907. The total attendance was fifty, including the following thirty-eight members of the Society :

Professor W. W. Beman, Mr. G. D. Birkhoff, Professor. O. Bolza, Mr. W. E. Brooke, Professor C. E. Comstock, Professor D. R. Curtiss, Professor E. W. Davis, Professor L. E. Dickson, Dr. E. L. Dodd, Professor L. W. Dowling, Mr. E. P. R. Duval, Mr. E. B. Escott, Dr. Peter Field, Professor A. G. Hall, Professor C. N. Haskins, Professor E. R. Hedrick, Mr. L. Ingold, Professor O. D. Kellogg, Dr. H. G. Keppel, Professor Kurt Laves, Mr. N. J. Lennes, Mr. W. D. MacMillan, Professor H. Maschke, Professor G. A. Miller, Professor E. H. Moore, Dr. J. C. Morehead, Professor F. R. Moulton, Dr. L. I. Neikirk, Mr. F. W. Owens, Professor D. A. Rothrock, Professor W. J. Rusk, Mr. A. R. Sch weitzer, Professor E. B. Skinner, Professor H. E. Slaught, Professor E. J. Townsend, Professor E. B. Van Vleck, Dr. A. E. Young, Professor J. W. A. Young.

The chairman of the section, Professor E. B. Van Vleck, called the meeting to order and presented Professor $\mathrm{H}$. Maschke, Vice-President of the Society, who presided at both the morning and afternoon sessions. It was voted that hereafter, at the discretion of the programme committee, two days be given to both the April and December meetings of the Section. A full meeting of the enlarged committee on programme for the December, 1907, meeting was held and definite plans were formulated for a symposium on the teaching of engineering mathematics.

The following papers were read :

(1) Dr. L. I. NeIKIRK : "A geometric representation of the Galois field."

(2) Mr. A. R. Schweitzer: "On the Ausdehnungslehre of Grassmann."

(3) Professor E. W. Davis: "A geometric construction for the imaginary intersections of a conic and a line" (preliminary communication). 
(4) Professor L. E. Dickson: "Representations of the general symmetric group as linear groups in finite and infinite fields."

(5) Professor E. B. Van Vleck: "Note on curves without tangents."

(6) Dr. A. E. Young: "Asymptotic and isothermic surfaces."

(7) Mr. Lovis INGOLd : "Vector theory in terms of symbolic differential parameters."

(8) Mr. E. B. EscotT: "New expressions for the number of classes of quadratic forms."

(9) Professor O. Bolza : " Existence proof for a field of extremals tangent to a given curve."

(10) Dr. Peter Field : "Note on a certain plane quintic curve."

(11) Professor L. E. Dickson : "The symmetric group on eight letters and the senary first hypoabelian group."

(12) Dr. C. H. Sisam : "On a property of parabolas and hyperbolas of $m$ th degree."

(13) Mr. LouIs INGOLI) : "Vector differential geometry in $n$-space."

(14) Dr. E. L. Dodd : "The iterated generalized limits of Cesàro and Borel."

(15) Mr. G. D. Binkнонғ : "Boundary value problems of ordinary linear differential equations."

(16) Professor G. A. Miller: "Groups defined by the orders of two operators and the order of their commutator."

(17) Professor O. Bolza : "Concerning conjugate points in the case of discontinuous solutions in the calculus of variations."

(18) Professor Kunt Laves: "Jacobi's partial differential equations, II."

(19) Professor R. D. Carmichael: "On certain quartic curves which may degenerate into an ellipse."

(20) Professor R. D. Carmichael: "A table of the numbers corresponding to given values of Euler's function $\phi(m)$."

(21) Professor R. D. Carmichael: "On constructing a cube having a given ratio to a given cube."

(22) Professor C. C. Grove: "The complete Pappus hexagon."

(23) Professor E. R. Hedrick : “On implicit functions" (preliminary communication). 
In the absence of the authors, the papers of Dr. Sisam and Professor Carmichael were read by title, Also, owing to the length of the programme, the second paper of Professor Bolza and the paper of Professor Hedrick were read by title.

Abstracts of the papers follow below. The abstracts are numbered to correspond to the titles listed above.

1. In this paper Dr. Neikirk gives a construction of the algebraic number systems of the form

$$
\omega=\sum_{r=1}^{r=n} C_{r} j^{n-r}
$$

where $j$ is a root of the irreducible equation $f(x)=0$ and the $C$ 's are integral. This was accomplished on the ordinary form of complex plane. The results were then applied to finite fields, such as the Galois field, by distinguishing two kinds of points ; $i$. e., reduced points and congruent points.

2. Mr. Schweitzer observes that his $n$-dimensional geometric $K_{n}$ system $*$ ( $\left.n \geqq 3\right)$ forms a convenient descriptive basis for the consideration of the fundamental principles of the Ausdehnungslehre. In fact, the statement $\alpha_{1} \alpha_{2} \cdots \alpha_{n+1} K_{n} \beta_{1} \beta_{2} \cdots \beta_{n+1}$ may be identified with the statement

$$
s\left(\alpha_{1} \alpha_{2} \cdots \alpha_{n+1}\right)=t\left(\beta_{1} \beta_{2} \cdots \beta_{n+1}\right),
$$

where $s$ and $t$ denote real numbers such that $s t>0$ and the indicated $(n+1)$-ads denote elementary magnitudes of the $(n+1)$ st grade.

3. Professor Davis calls the point $\left(x^{\prime}, y^{\prime}\right)$ a black point. It is joined by a red line to the point $\left(x^{\prime}+x^{\prime \prime}, y^{\prime}+y^{\prime \prime}\right)$ called a blue point. Thus $x^{\prime}$ and $y^{\prime}$ are the real parts of the coordinates, while $x^{\prime \prime}$ and $y^{\prime \prime}$ are the imaginary parts of the coordinates of the point $\left(x^{\prime}+i x^{\prime \prime}, y^{\prime}+i y^{\prime \prime}\right)$.

If there be a linear relation between $x^{\prime}+i x^{\prime \prime}$ and $y^{\prime}+i y^{\prime}$ then there is in general a $(1,1)$ correspondence set up between black points and blue points in the plane. But if the blue line joining two blue points be parallel to the black line joining the corresponding black points, then all the points satisfying the linear relation and no others are satisfied by the elements repre-

* Cf. Bulletin, Nov., 1906. 
sented by joining any point whatsoever of the black to any point whatsoever of the parallel blue line.

If the blue line coincide with the black line there results an ordinary real line.

4. The first paper by Professor Dickson gives a general investigation of the linear homogeneous groups on $m$ variables with coefficients in a field $F$ which are simply isomorphic with the symmetric group on $q$ letters. In particular, it is shown that $m \geqq q-1$, unless $F$ has a modulus dividing $q$. In the latter case there are representations on $q-2$ variables but not on fewer.

5. The paper of Professor Van Vleck, after briefly discussing a simple example of a continuous curve without a tangent, passed to the consideration of an example, simpler than any given hitherto, of a continuous curve which has an infinite number of oscillations in every part, however small, and yet possesses an ordinary tangent. This was followed by the following theorem : If in the interval $(a, b)$ and in every subinterval the continuous curve $y=\phi(x)$ is of unlimited variation, there is a set of points of the second category, everywhere dense upon the curve, at which $\phi(x)$ has no derivative. This theorem may be considered as the complement of one previously obtained by Lebesgue for a continuous curve of limited variation.

6. Dr. Young called attention to the fact that certain of the known isothermic surfaces have isothermal asymptotic lines and undertook in his paper to show how all such surfaces may be obtained. He showed that the simplest treatment of the problem was from the standpoint of the spherical representation of the lines of curvature. For the problem reduces to that of finding all isothermic surfaces for which the spherical images of the lines of curvature are isotherms. Dr. Young obtained both the singular and general solution of an equation which expressed the condition of integrability of the Codazzi equations, and in this way reduced the problem to that of determining those forms of the arbitrary functions, thus obtained, which will satisfy the Gauss equation for the sphere. The singular solution leads to the quadrics, certain cyclides, certain surfaces associated with these, which Dr. Young has discussed in a previous paper, and finally to a number of new surfaces whose cartesian coordinates he has obtained. The latter furnish a clew 
to two new sets of isothermic surfaces of which the surfaces found are particular cases. These be will report upon later.

The most general case arising from the general and singular solution combined Dr. Young has not completed, but he has carried the work far enough to indicate final success.

7. In his first paper Mr. Ingold exhibits a theory of vectors in a euclidean $n$-space $S_{n}$, as a special chapter of Professor Maschke's symbolic differental invariant theory. The derivatives $f_{1}, \cdots, f_{n}$ of the symbolic function $f$ are identified with a set of $n$ mutually orthogonal unit vectors of $S_{n}$; equivalent reference systems are introduced and symbolic expressions are obtained for $\lambda$-dimensional forms in $S_{n}$, inner and outer products, product determinant expansions, and regressive products. Finally the symbols of a $\lambda$-space $A_{\lambda}$ contained in $S_{n}$ are identified with a system of vectors tangent to the parameter curves of $A_{\lambda}$.

8. Lejeune Dirichlet has given expressions for the number of classes of forms of negative determinants $D=-P$ or $-2 P$ in terms of the sum of the Jacobi symbols $S / P$ for the octants and twelfths of $P$, and Dedekind has shown in his notes on Gauss's Works (Untersuchungen über höhere Arithmetik, pages 673-7, 693-5) how from these formulas Gauss's expressions for the distribution of the quadratic residues in the octants and twelfths of $P$ may be obtained. In Cielle's Journal, volume 127 (1904), pages 1-19, Karpinski has extended the method of Dedekind to find the distribution of the quadratic residues in the 10 ths and 24 ths of $P$.

In the present paper, Mr. Escott has generalized the result of Dr. Karpinski for the division of $P$ into $u$ ths and has shown how these formulas will simplify in many cases the finding of the number of classes for a given determinant.

9. In his paper on "Sufficient condition for a minimum with respect to one-sided variations" (Transactions, volume 5, pp. 477-492) Professor Bliss has proved that under certain conditions a set of extremals tangent to a given curve form a field. This proof is indirect inasmuch as he proves the theorem first for the special case of curves representable in the form $y=f(x)$, and then reduces the general case of parameter representation to the former by a point transformation of the plane. The object of Professor Bolza's first note is to give a 
direct proof of the theorem for the general case of parameter representation.

10. In a paper published in the last volume of the Quarterly Journal of Mathematics Mr. Bassett obtains the equation of a rational quintic curve having four cusps, from the fact that it is the dual of a rational quartic having one cusp. Dr. Field derived the equation and general form of the curve by inverting a tricuspid quartic which passes through the vertices and is tangent to one side of the triangle of reference.

11. Professor Dickson's second paper appeared in full in the May Bulletin.

12. Dr. Sisam derived a formula for the number of tangents to an arbitrary algebraic curve such tha he tangents at two intersections of any one of them with the urve intersect on the curve. He then showed that when the given curve is a parabola or hyperbola of $m$ th degree, the tangents at any pair of points lying on the same tangent meet on the curve, and conversely. Several interesting properties of the correspondences thus established between the points and tangents of the curve were also discussed.

13. Mr. Ingold's second paper contains generalizations of the ordinary vector differential geometry as given by BuraliForti and Fehr. A system of vectors tangent to the parameter lines is taken as reference system on an $(n-1)$-dimensional surface : these are determined from the cartesian representation by the methods of the first paper.

14. The iterated generalized limits of a multiple sequence may be unequal. Moreover, the existence of iterated generalized limits does not necessitate the existence of the generalized limit of a multiple sequence, nor vice versa. Dr. Dodd extends his treatment of iterated limits (Mathematische Annalen, volume 61, Heft 1) to the generalized limits of Cesàro and Borel.

15. The fundamental theorem of Mr. Birkhoff's paper is concerned with the character of the solutions of a differential equation

$$
\frac{d^{n} z}{d x^{n}}+\rho a_{n-1}(x, \rho) \frac{d^{n-1} z}{d x^{n-1}}+\cdots+\rho^{n} a_{0}(x, \rho) z=0
$$


for large values of the complex parameter $\rho, a \leqq x \leqq b$. Here for $|\rho| \geqq R$

$$
a_{i}(x, \rho)=\sum_{j=0}^{\infty} a_{i j}(x) \cdot \frac{1}{\rho} j .
$$

The theorem holds on certain regions, $\theta \leqq \arg \rho \leqq \phi$, of the $\rho$-plane. With its aid is solved in large measure the problem to determine the distribution of those values of $\rho$ for which a solution of (1) exists fulfilling $n$ conditions

(2) $\sum_{j=0}^{n-1}\left\{\alpha_{i j}(\rho) \frac{d^{j} z}{d x^{j}}(a, \rho)+\beta_{i j}(\rho) \frac{d^{j} z}{d x^{j}}(b, \rho)\right\}=0 \quad(i=1,2, \ldots, n)$, and to determine the character of these solutions. Here for $|\rho| \geqq R$

$$
\alpha_{i j}(\rho)=\sum_{k=0}^{\infty} \alpha_{i j k} \cdot \frac{1}{\rho} k ; \quad \beta_{i j}(\rho)=\sum_{k=0}^{\infty} \beta_{i j l} \cdot \frac{1}{\rho} k .
$$

Using these facts, theorems analogous to those which Kneser has given in the case $n=2$ are derived concerning the expansion of a function $f(x)$ in a series of solutions of a differential equation and conditions (like (1), (2))

$$
\begin{aligned}
& p_{n}(x) \frac{d^{n} z}{d x^{n}}+p_{n-1}(x) \frac{d^{n-1} z}{d x^{n-1}}+\cdots+p_{0}(x) z+\rho^{n} A(x) z=0, \\
& \sum_{j=0}^{n-1}\left\{\alpha_{i j} \frac{d^{j} z}{d x^{j}}(a, \rho)+\beta_{i j} \frac{d^{j} z}{d x^{j}}(b, \rho)\right\}=0 \quad(i=1,2, \cdots, n)
\end{aligned}
$$

in the real self- or opposite-adjoint case, $p_{n}(x) \neq 0, A(x) \neq 0$. It is also proved that this restriction to the real self- or oppositeadjoint case are to a great extent unessential.

16. The object of Professor Miller's paper is to develop theorems which will make the commutator a more useful instrument in the study of group properties. The results are analogous to those which relate to the groups of genus zero. The following theorems are proved: Any dihedral group whose order is divisible by 8 may be defined by the orders of two generators and the order of the commutator of these generators, and no other groups are known to have this property. There is an infinite system of groups such that each of them is generated by two operators whose commutator is of order 2 while the order of one of the generators is any given number which 
exceeds 3 and the order of the other is any given even number greater than zero. If the orders of the two generators of a group are either 3,4 or 3,5 and if the order of their commutator is 2 , these generators may be so selected as to give rise to an arbitrary group in an infinite system. If a group is generated by two operators of orders 2 and 3 respectively whose commutator is of order 3, its commutator subgroup is generated by two operators of order 3 whose product is also of this order. There are three and only three such groups which have an abelian commutator subgroup. The orders of these groups are 6,18, and 54 respectively.

17. Professor Bolza's second note supplements Dr. Carathéodory's investigation concerning conjugate points for discontinuous solutions in the calculus of variations, by giving in explicit form the equation which connects the parameters of a pair of such conjugate points.

18. In a previous paper by Professor Laves on the same subject (presented at the Chicago meeting of April, 1906) it was shown that the partial differential equation for a body rotating about a fixed point can be integrated by determining the action of the system between two terminal positions. It is here shown how this proceeding rests on a theorem of Jacobi for problems depending upon two parameters. A family of orthogonal curves, one set of which are the extremals for the same value of the constant of energy, while the other are the transversals, covers the plane. The action of the system between two positions $A_{0}$ and $A_{1}$ is the difference between the values of the transversals passing through these same points. This is the form given to Jacobi's theorem by Sir William Thomson.

19. Professor Carmichael's first paper appeared in full in the March number of the American Mathematical Monthly.

20. In this table Professor Carmichael gives every possible value of Euler's function $\phi(m)$ up to 1000 , and lists opposite each value all the numbers $m$ which correspond to it. It is now more than 30 years since Cayley expressed a desire for the construction of this table. It will, it is believed, be found useful in various ways in the study of number theory.

21. In his third paper Professor Carmichael constructs the semicubical parabola by continuous motion, and applies the 
locus to finding a cube having to a given cube any ratio which may be expressed by two straight lines.

22. The proposition, "If $A, B, C$ are three points of one line and $A^{\prime}, B^{\prime}, C^{\prime}$ are three points of another line, then the intersections, $B C^{\prime} / B^{\prime} C, C A^{\prime} / C^{\prime} A, A B^{\prime} / A^{\prime} B$ lie on a line" (Salmon : Conics, $\S 268$ ) puts in simple form Propositions 138, 139, Liber VII, Collectiones of Pappus. The figure arising is a special case of the Pascal hexagon which may be called the Pappus hexagon. Professor Grove's paper develops the complete Pappus hexagon and shows the duality of the figure in the following and other theorems with their duals:

I. Three points $\pi_{i}$ on each of two lines $D_{1}, D_{2}$ meeting in the point $\delta_{1}$ are cross-joined by nine lines which meet in 18 points $1-18$, which are the vertices of two sets of three line triads each. The triangles of each set are inter se in triple perspective, having as axes of perspective the lines $D_{1}$ and $D_{2}$ each three times and three lines $P_{i}$ on the point $\Sigma_{2}$ or $\Sigma_{1}$ respectively, and having as centers of perspective the point $\Sigma_{1}$ or $\Sigma_{2}$ three times each for the sets respectively and twelve other points all of which lie on a covariant line $E_{1}$ which is the polar of $\delta$ as to any of the six triangles. The 18 points lie by threes on six lines $P_{i}$, which are three and three on the points $\Sigma_{1}$ and $\Sigma_{2}$ above.

II. The points $\Sigma_{1}$ and $\Sigma_{2}$ and $\delta$ are the false vertices of the complete quadrangle of the Hessian pairs of the point triads $\pi_{i}$ on $D_{1}$ and on $D_{2}$.

Following names given in the complete Pascal hexagon, he calls the three points $\pi_{1}, \pi_{3}, \pi_{5}$ on $D_{1}$, and $\pi_{2}, \pi_{4}, \pi_{6}$ on $D_{2}$ Pappus vertices ; the three lines $P_{i}$ on $\Sigma_{1}$ and $\Sigma_{2}$ each, he calls Pappus lines; the points $\Sigma_{1}$ Steiner points whose line is $S_{i}$, the lines $D_{1}$ Hessian diagonals. Taking the lines $P_{i}$ from the above theorem and proceeding in the dual form, we get three points $\pi_{i}^{\prime}$ on each of the same two lines $D_{i}$ etc., etc. The derived triads $\pi_{i}^{\prime}$ and the counter-triads of the original triads are in an involution whose double points are the polar pair of the intersection $\delta$ as to the original triads $\pi_{i}$. Six other theorems follow which deal with special forms.

This paper will appear in full in the May number of the American Mathematical Monthly.

23. In this paper Professor Hedrick shows that the ordinary statements of the fundamental theorem on the existence of a solution of the implicit equation $F(x, y)=0$ and their demon- 
strations are included in the statement and demonstration of the more general and simple theorem :

If $F(x, y)=0$ is satisfied by $x=x_{0}, y=y_{0}$, and if $F(x, y)$ is a continuous function of $x$ and a continuously increasing (or decreasing) function of $y$ near $\left(x_{0}, y_{0}\right)$, then there exists one singlevalued solution $y=\phi(x)$ near $\left(x_{0}, y_{0}\right)$ such that $y_{0}=\phi\left(x_{0}\right)$ and $F(x, \phi(y))=0$; and that solution is continuous.

A discussion of the relation of this theorem to other forms was appended.

\author{
H. E. Staugut, \\ Secretary of the Chicago Section.
}

\title{
ON A LIMIT OF THE ROOTS OF AN EQUATION THAT IS INDEPENDENT OF ALL BUT TWO OF THE COEFFICIENTS.
}

BY PROFESSOR R. E. ALLARDICE.

(Read before the San Francisco Section of the American Mathematical Society, February 23, 1907.)

At the end of a paper by Dr. Landau,* it is shown that every equation of the form $a x^{n}+x+1=0$ has a root whose modulus is not greater than 2 , and that every equation of the form $a x^{n}+b x^{m}+x+1=0$ has a root whose modulus is not greater than 8 . The object of the present paper is to show that every equation of the form

$$
a x^{n}+b x^{m}+c x^{l}+\cdots+a_{1} x+a_{0}=0
$$

has a root whose modulus is not greater than

$$
\left|\frac{a_{0}}{a_{1}}\right| \cdot \frac{n}{n-1} \cdot \frac{m}{m-1} \cdot \frac{l}{l-1} \cdots
$$

whatever be the values of the coefficients $a, b, c, \ldots$; and that, for certain values of these coefficients, this limit is attained.

\footnotetext{
"Ueber den Picardschen Satz," Vierteljahrsschrift der Naturforschenden Gesellschaft in Zürich, Jahrgang 51, 1906.
} 